\title{
Convergence results for unbounded solutions of first order non-linear differential-functional equations
}

\author{
by HeNRYK LESZCZYŃSKi (Gdańsk)
}

\begin{abstract}
We consider the Cauchy problem in an unbounded region for equations of the type either $D_{t} z(t, x)=f\left(t, x, z(t, x), z_{(t, x)}, D_{x} z(t, x)\right)$ or $D_{t} z(t, x)=f(t, x, z(t, x)$, $\left.z, D_{x} z(t, x)\right)$. We prove convergence of their difference analogues by means of recurrence inequalities in some wide classes of unbounded functions.
\end{abstract}

Introduction. Basic uniqueness results for first order differential equations were proved by Szarski [8], and then generalized by Kamont [3], Besala [1] and others. Let us mention [6] where the case of differential-functional equations was treated.

Uniqueness, existence and convergence results for parabolic equations require some assumptions on the class of solutions, namely one ought to assume that the solutions and their derivatives grow at most as exp $\left(c\|x\|^{2}\right)$ (see [4]). The convergence of difference schemes was proved first locally, next in the unbounded case for differential problems [2], and finally for differential-functional systems using a special type of difference operators [7], and with general difference analogues consistent with the differentialfunctional problem [5].

We extend general methods of proving convergence by means of difference inequalities described in [8] and in the references mentioned there. Working in wide functional classes (see [6]), we prove recurrence estimates in a way similar to that used for parabolic equations. We deal simultaneously with two main types of functional dependence: first, with the variable $z_{(t, x)}$ as an extension of retardations and integrations over a rectangular bounded left-side neighbourhood of the point $(t, x)$, and secondly, with $z$ appearing as variable in a function of the Volterra type. These two quite general models of functional dependence coincide in classes of bounded solutions; however, if we investigate unbounded functions, then two slightly different sets of as-

1991 Mathematics Subject Classification: Primary 35A35; Secondary 35 B35.

Key words and phrases: error estimates, recurrence inequalities, difference scheme. 
sumptions imply uniqueness and convergence as shown in [6]. Finally, we remark that our results, formulated for one equation, can easily be proved for weakly coupled systems.

1. Basic notations and formulation of the first differential-functional problem. Let $E_{0}=\left[-\tau_{0}, 0\right] \times \mathbb{R}^{n}, E=[0, a] \times \mathbb{R}^{n}$ and $D=\left[-\tau_{0}, 0\right] \times$ $[-\tau, \tau]$, where $\tau_{0} \in \mathbb{R}_{+}, a>0$ and $\tau=\left(\tau_{1}, \ldots, \tau_{n}\right) \in \mathbb{R}_{+}^{n}$. If $z \in C\left(E_{0} \cup E, \mathbb{R}\right)$ and $(t, x) \in E$, then $z_{(t, x)}: D \rightarrow \mathbb{R}$ is defined by $z_{(t, x)}(\bar{t}, \bar{x})=z(t+\bar{t}, x+\bar{x})$ for $(\bar{t}, \bar{x}) \in D$.

A function $H$ is of class $\mathcal{H}$ iff $H \in C\left(E_{0} \cup E,(0, \infty)\right)$, the functions $H_{\mid E_{0}}$ and $H_{\mid E}$ are continuously differentiable, and

(i) $H(\cdot, x)$ is non-decreasing for every $x \in \mathbb{R}^{n}$,

(ii) $x_{i} D_{x_{i}} H(t, x) \geq 0$ for $i=1, \ldots, n$ and $(t, x) \in E_{0} \cup E$, where $x=$ $\left(x_{1}, \ldots, x_{n}\right)$.

If $H \in \mathcal{H}$, then a function $\varepsilon$ is said to be of class $\mathcal{E}_{H}$ iff $\varepsilon \in C\left(E_{0} \cup\right.$ $E,(0, \infty)), \varepsilon(t, x) \leq \varepsilon(\bar{t}, \bar{x})$ for $\|x\| \geq\|\bar{x}\|, 0 \leq t, \bar{t} \leq a$, and the function $\widetilde{H}$ defined by $\widetilde{H}(t, x)=H(t, x) \varepsilon(t, x)$ for $(t, x) \in E_{0} \cup E$ belongs to $\mathcal{H}$.

A function $z$ is of class $\mathcal{C}_{H}$ (resp. $\mathcal{C}_{H, \varepsilon}$ ) iff $z \in C\left(E_{0} \cup E, \mathbb{R}\right.$ ) and $|z(t, x)| / H(t, x)$ (resp. $|z(t, x)| / \widetilde{H}(t, x)$, where $\widetilde{H}(t, x)=H(t, x) \varepsilon(t, x))$ is bounded.

The classes $\mathcal{C}_{H}$ and $\mathcal{C}_{H, \varepsilon}$ are equipped with the seminorms $\|\cdot\|_{H}(t)$ defined by

$$
\|z\|_{H}(t)=\sup \left\{|z(\bar{t}, \bar{x})| / H(\bar{t}, \bar{x}) \mid(\bar{t}, \bar{x}) \in E_{0} \cup E, \bar{t} \leq t\right\}
$$

for $(t, x) \in(0, a]$ and $z \in \mathcal{C}_{H}$. The space $C(D, \mathbb{R})$ is equipped with the maximum norm $\|\cdot\|_{D}$. Denote by $\Omega^{(0)}$ and $\Omega_{H}^{(1)}$ the sets

$$
\Omega^{(0)}=E \times \mathbb{R} \times C(D, \mathbb{R}) \times \mathbb{R}^{n} \quad \text { and } \quad \Omega_{H}^{(1)}=E \times \mathbb{R} \times \mathcal{C}_{H} \times \mathbb{R}^{n} .
$$

If $\mathcal{L} \in C\left(E_{0} \cup E, \mathbb{R}_{+}\right)$and $L_{1}, L_{2} \in \mathbb{R}_{+}$, then a function $f$ is of class $\operatorname{Lip}\left(\Omega^{(0)} ; \mathcal{L}, L_{1}, L_{2}\right)$ iff $f \in C\left(\Omega^{(0)}, \mathbb{R}\right)$ and

$$
\begin{aligned}
& |f(t, x, p, w, q)-f(t, x, \bar{p}, \bar{w}, \bar{q})| \\
& \quad \leq L_{1}|p-\bar{p}|+\mathcal{L}(t, x)\|w-\bar{w}\|_{D}+L_{2}\|q-\bar{q}\|
\end{aligned}
$$

for all $(t, x, p, w, q),(t, x, \bar{p}, \bar{w}, \bar{q}) \in \Omega^{(0)}$.

Now, let $f \in C\left(\Omega^{(0)}, \mathbb{R}\right)$. We consider the differential-functional equation

$$
D_{t} z(t, x)=f\left(t, x, z(t, x), z_{(t, x)}, D_{x} z(t, x)\right),
$$

where $D_{x} z(t, x)=\left(D_{x_{1}} z(t, x), \ldots, D_{x_{n}} z(t, x)\right)$, with the initial condition

$$
z(t, x)=\phi(t, x), \quad(t, x) \in E_{0},
$$

where $\phi \in C\left(E_{0}, \mathbb{R}\right)$. 
We assume throughout the paper that the Cauchy problem (1.3), (1.4) has a unique solution of class $\mathcal{C}_{H}$ defined on $E_{0} \cup E$ (see [6]).

Let $\Gamma: \mathbb{R}_{+} \rightarrow \mathbb{R}_{+}$and $\kappa, \psi:\left[-\tau_{0}, a\right] \rightarrow(0, \infty)$ be continuously differentiable functions such that $\kappa^{\prime}(t) \geq 0$ and $\psi^{\prime}(t) \geq 0$ for $t \in[0, a]$; $\kappa^{\prime}(t)=\psi^{\prime}(t)=0$ for $t \in\left[-\tau_{0}, 0\right]$; and $\Gamma^{\prime}(t) \geq 0$ for $t \in \mathbb{R}_{+}$.

We define

$$
H(t, x)=\Gamma\left(\psi(t) \sqrt{1+\|x\|^{2}}\right)
$$

for $(t, x) \in E_{0} \cup E$, and

$$
\mathcal{L}(t, x)=\bar{p} / \Gamma\left(\kappa(t) \sqrt{1+\|x\|^{2}}\right)
$$

for $(t, x) \in E$, where $\bar{p} \in \mathbb{R}_{+}$. Some conditions, assumed in [6], on the functions $\Gamma, \kappa, \psi$ imply uniqueness for problem (1.3), (1.4) as well as for the Cauchy problem with another type of functional dependence. The properties of these functions assumed in the present paper are very close to those in [6].

EXAMPLE. Let us list a few examples of functions $\Gamma$ appearing in (1.5), (1.6):

(i) $\Gamma(t)=t^{k}$ for $t \in \mathbb{R}_{+}$and $k>0$.

(ii) $\Gamma(t)=\exp \left(t^{m}\right)$ for $t \in \mathbb{R}$ and $m \in \mathbb{N}$.

(iii) $\Gamma(t)=e_{l}(t)$ for $t \in \mathbb{R}_{+}$and $l \in \mathbb{N}$, where $e_{0}(t)=t$ and $e_{l+1}(t)=$ $\exp \left(e_{l}(t)\right)$ for $l=0,1, \ldots$ and $t \in \mathbb{R}$.

(iv) $\Gamma(t)=\Gamma^{\mathrm{T}}(t)=\exp \left(\int_{0}^{t} \widetilde{\Gamma}(r+1) d r\right)$ for $t \in \mathbb{R}$, where $\widetilde{\Gamma}(r)=$ $(r-n) e_{n+1}(n+1)+(n+1-r) e_{n}(n)$ for $r \in[n, n+1]$ and $n=0,1, \ldots$ This example shows that $\Gamma$ can grow faster than all $e_{l}$ for $l=0,1, \ldots$ It is possible to construct still faster growing functions:

(v) $\Gamma(t)=\Gamma_{, i}^{\mathrm{T}_{i}}(t)$ for $t \in \mathbb{R}_{+}$and $i=0,1, \ldots$, where $\Gamma_{, 0}^{\mathrm{T}}(t)=\Gamma^{\mathrm{T}}(t)$ and $\Gamma_{, k+1}^{\mathrm{T}}(t)=\exp \left(\Gamma_{, k}^{\mathrm{T}}(t)\right)$ for $k=0,1, \ldots$; and next:

(vi) $\Gamma(t)=\Gamma^{\mathrm{TT}_{(}}(t)=\exp \left(\int_{0}^{t} \widetilde{\Gamma}(r+1) d r\right)$ for $t \in \mathbb{R}_{+}$, where $\widetilde{\Gamma}(r)=$ $(r-l) \Gamma_{, l+1}^{\mathrm{T}}(l+1)+(l+1-r) \Gamma_{, l}^{\mathrm{T}}(l)$ for $r \in[l, l+1]$ and $l=0,1, \ldots$, and so on.

(vii) If $\xi \in C(\mathbb{R}, \mathbb{R})$ and

$$
\omega(t)=\exp \left(-\max \left\{0,(1-t)^{-1}\right\}\right)\left(\int_{1}^{\infty} e^{-s} s^{-3 / 2}(s-1)^{-1 / 2} d s\right)^{-1}
$$

for $t \in \mathbb{R}$, then

$$
\Gamma(t)=C_{0} \exp \left(\int_{-\infty}^{\infty} \max _{s \in[-r-1, r+1]}|\xi(s)| \omega\left(\sqrt{1+t^{2}}-r\right) d r\right), \quad t \in \mathbb{R}_{+},
$$

where $C_{0}=1+\max \{|\xi(r)| \mid r \in[-1,1]\}$, is differentiable and satisfies $\Gamma(|t|) \geq|\xi(t)|$ for $t \in \mathbb{R}$. 
3. Formulation of the difference problem and consistency lemmas. Let $\bar{h}=\left(\bar{h}_{0}, \bar{h}^{\prime}\right) \in(0, \infty)^{1+n}$, where $\bar{h}^{\prime}=\left(\bar{h}_{1}, \ldots, \bar{h}_{n}\right)$, and $I_{d}$ be a non-empty subset of

$$
\left\{\begin{array}{l|l}
h=\left(h_{0}, h^{\prime}\right) \in \mathbb{R}_{+}^{1+n} & \begin{array}{l}
h^{\prime}=\left(h_{1}, \ldots, h_{n}\right) ; h_{i} \in\left(0, \bar{h}_{i}\right], i=1, \ldots, n ; \\
h_{0} N_{0}=\tau_{0} \text { for some } N_{0} \in\{0,1, \ldots\}
\end{array}
\end{array}\right\} .
$$

Let $(t, x)^{(\eta)}=\left(t^{(\eta)}, x^{(\eta)}\right)$, where $t^{(\eta)}=h_{0} \eta_{0}$ and $x^{(\eta)}=\left(h_{1} \eta_{1}, \ldots, h_{n} \eta_{n}\right)$ for $\eta=\left(\eta_{0}, \eta^{\prime}\right) \in \mathbb{Z}^{1+n}$ and $\eta=\left(\eta_{1}, \ldots, \eta_{n}\right)$. For $h=\left(h_{0}, h^{\prime}\right) \in I_{d}$ there is a natural constant $N_{*}$ and $N_{1}, \ldots, N_{n} \in \mathbb{Z}_{+}$such that $N_{*} h_{0} \leq a<\left(N_{*}+1\right) h_{0}$ and $\tau_{i} \leq N_{i} h_{i}<\tau_{i}+h_{i}$ for $i=1, \ldots, n$.

Let $h=\left(h_{0}, h^{\prime}\right) \in I_{d}$. Then we define

$$
\begin{aligned}
E_{0, h} & =\left\{(t, x)^{(\eta)} \mid \eta=\left(\eta_{0}, \eta^{\prime}\right) \in \mathbb{Z}^{1+n}, \eta_{0} \in\left\{-N_{0}, \ldots, 0\right\}\right\}, \\
E_{h} & =\left\{(t, x)^{(\eta)} \mid \eta=\left(\eta_{0}, \eta^{\prime}\right) \in \mathbb{Z}^{1+n}, \eta_{0} \in\left\{0, \ldots, N_{*}-1\right\}\right\}, \\
\widehat{E}_{h} & =\left\{(t, x)^{(\eta)} \mid \eta=\left(\eta_{0}, \eta^{\prime}\right) \in \mathbb{Z}^{1+n}, \eta_{0} \in\left\{-N_{0}, \ldots, N_{*}\right\}\right\}, \\
D_{h} & =\left\{(t, x)^{(\eta)} \mid \begin{array}{l}
\eta=\left(\eta_{0}, \eta^{\prime}\right) \in \mathbb{Z}^{1+n}, \eta_{0} \in\left\{-N_{0}, \ldots, 0\right\} ; \\
\eta^{\prime}=\left(\eta_{1}, \ldots, \eta_{n}\right) ;\left|\eta_{i}\right| \leq N_{i}, i=1, \ldots, n
\end{array}\right\} .
\end{aligned}
$$

Let $z_{h}$ be a function defined on $\widehat{E}_{h}$. Set $z_{h}^{(\eta)}=z_{h}\left(t^{(\eta)}, x^{(\eta)}\right)$ for $(t, x)^{(\eta)} \in \widehat{E}_{h}$. If $(t, x)^{(\eta)} \in E_{h}$ and $z_{h}$ is a function defined on $\widehat{E}_{h}$, then the function $\left(z_{h}\right)_{(\eta)}: D_{h} \rightarrow \mathbb{R}$ is defined by

$$
\left(z_{h}\right)_{(\eta)}\left((t, x)^{(\bar{\eta})}\right)=z_{h}\left((t, x)^{(\eta+\bar{\eta})}\right) \quad \text { for }(t, x)^{(\bar{\eta})} \in D_{h} .
$$

Denote by $\mathcal{F}\left(\widehat{E}_{h}, \mathbb{R}\right)$ the set of all functions from $\widehat{E}_{h}$ to $\mathbb{R}$. If $z \in C\left(E_{0} \cup E, \mathbb{R}\right)$, then $z_{h} \in \mathcal{F}\left(\widehat{E}_{h}, \mathbb{R}\right)$ denotes the restriction of $z$ to the mesh.

Let $\lambda \in\{1,2, \ldots\}$ and

$$
S_{\lambda}=\left\{s=\left(s_{1}, \ldots, s_{n}\right) \in \mathbb{Z}^{n}|| s_{i} \mid \leq \lambda, i=1, \ldots, n\right\} .
$$

We define the difference operators $A, \Delta_{0}$ and $\Delta=\left(\Delta_{1}, \ldots, \Delta_{n}\right)$ by

$$
\begin{aligned}
A z_{h}^{(\eta)} & =\sum_{s \in S_{\lambda}} a_{s}^{(\eta)} z_{h}^{\left(\eta_{0}, \eta^{\prime}+s\right)}, & & (t, x)^{(\eta)} \in E_{h}, \\
\Delta_{0} z_{h}^{(\eta)} & =h_{0}^{-1}\left(z_{h}^{\left(\eta_{0}+1, \eta^{\prime}\right)}-A z_{h}^{(\eta)}\right), & & (t, x)^{(\eta)} \in E_{h}, \\
\Delta_{l} z_{h}^{(\eta)} & =h_{l}^{-1} \sum_{s \in S_{\lambda}} b_{s, l}^{(\eta)} z_{h}^{\left(\eta_{0}, \eta^{\prime}+s\right)}, & & (t, x)^{(\eta)} \in E_{h}, l=1, \ldots, n,
\end{aligned}
$$

where $a_{s}^{(\eta)}$ and $b_{s, l}^{(\eta)}$ are real coefficients.

Assumption $H_{1}$. Suppose that the discrete operators $A$ and $\Delta$ defined by (2.1) satisfy the following conditions

(i) $\sum_{s \in S_{\lambda}} a_{s}^{(\eta)}=1, \sum_{s \in S_{\lambda}} a_{s}^{(\eta)} s_{l}=0$ for $l=1, \ldots, n$ and $(t, x)^{(\eta)} \in E_{h}$. 
(ii) $\sum_{s \in S_{\lambda}} b_{s, l}^{(\eta)}=0, \sum_{s \in S_{\lambda}} b_{s, l}^{(\eta)} s_{j}=\delta_{l j}$ for $j, l=1, \ldots, n$ and $(t, x)^{(\eta)}$ $\in E_{h}$.

(iii) there is $c \in \mathbb{R}_{+}$such that $\left|a_{s}^{(\eta)}\right|,\left|b_{s, l}^{(\eta)}\right| \leq c$ for $s \in S_{\lambda},(t, x)^{(\eta)} \in E_{h}$, $l=1, \ldots, n$ and $h \in I_{d}$.

(iv) there are constants $c_{0}, c_{1} \in(0, \infty)$ such that $c_{0} h_{0} \leq h_{l} \leq c_{1} h_{0}$ for $l=1, \ldots, n$.

This assumption is necessary to prove that the discrete operators $\Delta_{l}$ for $l=0,1, \ldots, n$ approximate the differential operators $D_{t}$ and $D_{x_{l}}$ for $l=1, \ldots, n$.

The operator $[\cdot]_{h}: \mathcal{F}\left(\widehat{E}_{h}, \mathbb{R}\right) \rightarrow C\left(E_{0} \cup E, \mathbb{R}\right)$ is defined by

$$
\left[z_{h}\right]_{h}(\bar{t}, \bar{x})=\sum_{r=-\lambda}^{0} \sum_{s \in S_{\lambda}} p_{r, s}^{(\eta)}(\bar{t}, \bar{x}) z_{h}^{\left(\eta_{0}+r, \eta^{\prime}+s\right)}
$$

for $z_{h}: \widehat{E}_{h} \rightarrow \mathbb{R}, t^{(\eta-\mathbf{1})}<\bar{t} \leq t^{(\eta)}$ and $x^{(\eta-\mathbf{1})}<\bar{x} \leq x^{(\eta)}$, where $(t, x)^{(\eta-\mathbf{1})},(t, x)^{(\eta)} \in \widehat{E}_{h}$ and $\mathbf{1}=(1, \ldots, 1) \in \mathbb{Z}^{1+n}$. If $(t, x)^{\left(\eta_{0}+r, \eta^{\prime}+s\right)} \notin \widehat{E}_{h}$, then $z_{h}^{\left(\eta_{0}+r, \eta^{\prime}+s\right)}$ means the same as $z_{h}^{\left(-N_{0}, \eta^{\prime}+s\right)}$.

Assumption $H_{2}$. Suppose that the functions $p_{r, s}^{(\eta)}: \mathbb{R}^{1+n} \rightarrow \mathbb{R}$ in formula (2.2) are bounded and satisfy

$$
\sum_{r=-\lambda}^{0} \sum_{s \in S_{\lambda}} p_{r, s}^{(\eta)}(\bar{t}, \bar{x})=1
$$

for $t^{(\eta-1)}<\bar{t} \leq t^{(\eta)}$ and $x^{(\eta-1)}<\bar{x} \leq x^{(\eta)}$, and

$$
\widehat{p}=\sup \left\{\begin{array}{l|l}
\sum_{r=-\lambda} \sum_{s \in S_{\lambda}}\left|p_{r, s}^{(\eta)}(\bar{t}, \bar{x})\right| & \begin{array}{c}
t^{(\eta-1)}<\bar{t} \leq t^{(\eta)} \\
x^{(\eta-1)}<\bar{x} \leq x^{(\eta)}, h \in I_{d}, h \\
(t, x)^{(\eta-1)},(t, x)^{(\eta)} \in \widehat{E}_{h} .
\end{array}
\end{array}\right\}<\infty
$$

Now, we define difference schemes which correspond to the differentialfunctional problem (1.3), (1.4). Let $f \in \mathcal{C}_{H}\left(\Omega^{(0)}\right)$. Then we consider the following difference-functional problem (cf. (1.3)):

$$
\Delta_{0} z_{h}^{(\eta)}=f\left((t, x)^{(\eta)}, z_{h}^{(\eta)},\left(\left[z_{h}\right]_{h}\right)_{(\eta)}, \Delta z_{h}^{(\eta)}\right), \quad(t, x) \in E_{h},
$$

with the initial condition

$$
z_{h}^{(\eta)}=\bar{\phi}_{h}^{(\eta)}, \quad(t, x) \in E_{0, h} .
$$

In the literature the function $f$ is often replaced by a function $f_{h}$ which is defined by use of a finite Taylor expansion of $f$. This way one can obtain better difference approximations to the differential problem.

Let $H \in \mathcal{H}$ and $\varepsilon \in \mathcal{E}_{H}$. A function $z_{h}$ is of class $\mathcal{F}_{H}^{(h)}$ (resp. of class $\left.\mathcal{F}_{H, \varepsilon}^{(h)}\right)$ iff there is a function $\bar{z} \in \mathcal{C}_{H}$ (resp. $\bar{z} \in \mathcal{C}_{H, \varepsilon}$ ) such that $\bar{z}_{h}=z_{h}$, where $h \in I_{d}$. 
Now, we prove an auxiliary lemma on consistency.

Lemma 2.1. Suppose that $H \in \mathcal{H}$ and Assumptions $H_{1}$ and $H_{2}$ are satisfied. Let $f \in \mathcal{C}_{H}\left(\Omega^{(0)}\right)$ with constants $L_{0}, L_{1}, L_{2} \in \mathbb{R}_{+}$and $\mathcal{L} \in C\left(E, \mathbb{R}_{+}\right)$. Let $u \in C\left(E_{0} \cup E, \mathbb{R}\right)$ be a solution of $(1.3)$, (1.4) such that $u, D_{t} u, D_{x_{l}} u \in$ $\mathcal{C}_{H}$ for $l=1, \ldots, n$, and there is $L_{u} \in \mathbb{R}_{+}$such that

$$
\begin{aligned}
\mid D_{x_{l}} u(t, x)- & D_{x_{l}} u(\bar{t}, \bar{x})|+| D_{t} u(t, x)-D_{t} u(\bar{t}, \bar{x}) \mid \\
& \leq L_{u}\left(|t-\bar{t}|+\sum_{j=1}^{n}\left|x_{j}-\bar{x}_{j}\right|\right) \max \{H(t, x), H(\bar{t}, \bar{x})\}
\end{aligned}
$$

for $(t, x),(\bar{t}, \bar{x}) \in E$ and $l=1, \ldots, n$. Then

$$
\left|\Delta_{0} u_{h}^{(\eta)}-f\left((t, x)^{(\eta)}, u_{h}^{(\eta)},\left(\left[u_{h}\right]_{h}\right)_{(\eta)}, \Delta z_{h}^{(\eta)}\right)\right| \leq \mu_{h}^{(\eta)},
$$

where

$$
\begin{aligned}
\mu_{h}^{(\eta)}= & h_{0} L_{u} H_{h}^{\left(\eta_{0}+1, \eta^{\prime}\right)}+h_{0}^{-1} \widehat{a} \lambda^{2}\left(\sum_{l=1}^{n} h_{l}\right)^{2} L_{u} H_{h}^{\left(\eta_{0},\left|\eta^{\prime}\right|+\lambda^{\prime}\right)} \\
& +\mathcal{L}\left((t, x)^{(\eta)}\right) H_{h}^{\left(\eta_{0},\left|\eta^{\prime}\right|+\lambda^{\prime}+1^{\prime}\right)} \widehat{p} \\
& \times\left(h_{0} \lambda\left\|D_{t} u\right\|_{H}(a)+(\lambda+1) \sum_{l=1}^{n} h_{l}\left\|D_{x_{l}} u\right\|_{H}(a)\right) \\
& +L_{2}\left\|\left(h_{1}^{-1}, \ldots, h_{n}^{-1}\right)\right\| \widehat{b}\left(\sum_{l=1}^{n} h_{l}\right)^{2} \lambda^{2} L_{u} H_{h}^{\left(\eta_{0},\left|\eta^{\prime}\right|+\lambda^{\prime}\right)},
\end{aligned}
$$

where $\lambda^{\prime}=(\lambda, \ldots, \lambda) \in \mathbb{Z}^{n}, 1^{\prime}=(1, \ldots, 1) \in \mathbb{Z}^{n}$, and

$$
\widehat{a}=\sup _{\eta} \sum_{s \in S_{\lambda} \backslash\left\{0^{\prime}\right\}}\left|a_{s}^{(\eta)}\right|, \quad \widehat{b}=\sup _{l, \eta} \sum_{s \in S_{\lambda} \backslash\left\{0^{\prime}\right\}}\left|b_{s, l}^{(\eta)}\right| .
$$

Proof. First, using the mean value theorem we have

$$
\begin{aligned}
& \left|D_{t} u\left((t, x)^{(\eta)}\right)-\Delta_{0} u_{h}^{(\eta)}\right| \\
& =\mid D_{t} u\left((t, x)^{(\eta)}\right)-h_{0}^{-1}\left\{u_{h}^{(\eta)}+h_{0} D_{t} u\left((t, x)^{(\eta)}+\theta_{0}^{(\eta)}(h)\right)\right. \\
& \left.\quad-\sum_{s \in S_{\lambda}} a_{s}^{(\eta)}\left(u_{h}^{(\eta)}+\sum_{l=1}^{n} h_{l} s_{l} D_{x_{l}} u\left((t, x)^{(\eta)}+\theta_{s}^{(\eta)}(h)\right)\right)\right\} \mid
\end{aligned}
$$

for $(t, x)^{(\eta)} \in E_{h}$, where

$$
\begin{aligned}
& \theta_{0}^{(\eta)}(h) \in\left(0, h_{0}\right) \times\left\{0^{\prime}\right\}, \\
& \theta_{s}^{(\eta)}(h) \in\{0\} \times\left[-\lambda h_{1}, \lambda h_{1}\right] \times \ldots \times\left[-\lambda h_{n}, \lambda h_{n}\right]
\end{aligned}
$$

for $s \in S_{\lambda}$. 
From (2.7), (2.11) and Assumption $H_{1}$ we obtain

$$
\begin{aligned}
& \left|D_{t} u\left((t, x)^{(\eta)}\right)-\Delta_{0} u_{h}^{(\eta)}\right| \\
& \quad \leq L_{u} h_{0} H_{h}^{\left(\eta_{0}+1, \eta^{\prime}\right)}+h_{0}^{-1} \widehat{a} \lambda^{2}\left(\sum_{l=1}^{n} h_{l}\right)^{2} L_{u} H_{h}^{\left(\eta_{0},\left|\eta^{\prime}\right|+\lambda^{\prime}\right)},
\end{aligned}
$$

where $\widehat{a}$ is defined by (2.10). In a similar way we obtain

$$
\left|D_{x_{l}} u\left((t, x)^{(\eta)}\right)-\Delta_{l} u_{h}^{(\eta)}\right|
$$

$$
=\left|D_{x_{l}} u\left((t, x)^{(\eta)}\right)-h_{l}^{-1} \sum_{s \in S_{\lambda}} b_{s, l}^{(\eta)}\left(u_{h}^{(\eta)}+\sum_{l=1}^{n} h_{l} s_{l} D_{x_{l}} u\left((t, x)^{(\eta)}+\theta_{s}^{(\eta)}(h)\right)\right)\right|
$$

for $(t, x)^{(\eta)} \in E_{h}$ and $l=1, \ldots, n$, and thus

$$
\left|D_{x_{l}} u\left((t, x)^{(\eta)}\right)-\Delta_{l} u_{h}^{(\eta)}\right| \leq h_{l}^{-1} \widehat{b}\left(\sum_{l=1}^{n} h_{l}\right)^{2} \lambda^{2} L_{u} H_{h}^{\left(\eta_{0},\left|\eta^{\prime}\right|+\lambda^{\prime}\right)}
$$

for $(t, x)^{(\eta)} \in E_{h}$ and $l=1, \ldots, n$. If $(\bar{t}, \bar{x}) \in E_{0} \cup E$ and $t^{(\eta-1)}<\bar{t} \leq t^{(\eta)}$, $x^{(\eta-1)}<\bar{x} \leq x^{(\eta)}$, where $(t, x)^{(\eta-1)},(t, x)^{(\eta)} \in \widehat{E}_{h}$, then

$$
\begin{aligned}
& \left|\left[u_{h}\right]_{h}(\bar{t}, \bar{x})-u(\bar{t}, \bar{x})\right| \\
& =\mid \sum_{r=-\lambda}^{0} \sum_{s \in S_{\lambda}} p_{r, s}^{(\eta)}(\bar{t}, \bar{x})\left(u(\bar{t}, \bar{x})+\left(t^{\left(\eta_{0}+r, \eta^{\prime}+s\right)}-\bar{t}\right) D_{t} u\left(\theta_{r, s}^{(\eta)}(\bar{t}, \bar{x}, h)\right)\right. \\
& \left.\quad+\sum_{l=1}^{n}\left(x^{\left(\eta_{0}+r, \eta^{\prime}+s\right)}-\bar{x}_{l}\right) D_{x_{l}} u\left(\theta_{r, s}^{(\eta)}(\bar{t}, \bar{x}, h)\right)\right)-u(\bar{t}, \bar{x}) \mid \\
& \leq \hat{p}\left(\left\|D_{t} u\right\|_{H}(a) \lambda h_{0}+\sum_{l=1}^{n} h_{l}(\lambda+1)\left\|D_{x_{l}} u\right\|_{H}(a)\right) H_{h}^{\left(\eta_{0},\left|\eta^{\prime}\right|+\lambda^{\prime}\right)},
\end{aligned}
$$

where $\widehat{p}$ is defined by $(2.4)$ and $\theta_{r, s}^{(\eta)}(\bar{t}, \bar{x}, h)$ is an intermediate point between $(t, x)^{\left(\eta_{0}+r, \eta^{\prime}+s\right)}$ and $(\bar{t}, \bar{x})$.

Let $(t, x) \in E$. From (2.16) we have

$$
\begin{aligned}
& \left\|\left(\left[u_{h}\right]_{h}\right)_{(t, x)}-u_{(t, x)}\right\|_{D} \\
& \quad=\max _{(\bar{t}, \bar{x}) \in D}\left\|\left[u_{h}\right]_{h}(t+\bar{t}, x+\bar{x})-u(t+\bar{t}, x+\bar{x})\right\| \\
& \quad \leq \widehat{p}\left(\left\|D_{t} u\right\|_{H}(a) \lambda h_{0}+\sum_{l=1}^{n} h_{l}(\lambda+1)\left\|D_{x_{l}} u\right\|_{H}(a)\right) H_{h}^{\left(\widetilde{\eta_{0}}, \widetilde{\eta}^{\prime} \mid+\lambda^{\prime}\right)},
\end{aligned}
$$

where $\left(\widetilde{\eta}_{l}-1\right) h_{l}<x_{l} \leq \widetilde{\eta}_{l} h_{l}$ for $l=0, \ldots, n$. Condition (2.8) follows from (2.13), (2.15) and (2.17). This finishes the proof. 
3. Convergence theorem. In this section we will prove that natural assumptions imply the convergence of the difference scheme (2.5), (2.6).

Theorem 3.1. Suppose that

1) $H \in \mathcal{H}$ is given by (1.5), and $\mathcal{L}$ is defined by (1.6), where $\kappa(t)>$ $\psi(t)>0$ for $t \in\left[-\tau_{0}, a\right], \psi, \kappa$ are increasing on $[0, a]$, and $f \in \mathcal{C}_{H}\left(\Omega^{(0)}\right)$ with $L_{1}, L_{2} \in \mathbb{R}_{+}$,

2) $u \in C\left(E_{0} \cup E, \mathbb{R}\right)$ is a solution of (1.3), (1.4) such that $u, D_{t} u, D_{x_{l}} u \in$ $\mathcal{C}_{H}$ for $l=1, \ldots, n$, and there is $L_{u} \in \mathbb{R}_{+}$such that condition (2.7) is satisfied,

3) $v_{h} \in \mathcal{F}\left(\widehat{E}_{h}, \mathbb{R}\right)$ is a solution of $(2.5),(2.6)$,

4) the following monotonicity condition is satisfied:

$$
a_{s}^{(\eta)}+h_{0} \sum_{l=1}^{n} h_{l}^{-1} b_{s, l}^{(\eta)} D_{q_{l}} f\left(P^{(\eta)}\right) \geq 0
$$

for $s \in S_{\lambda},(t, x)^{(\eta)} \in E_{h}$ and $P^{(\eta)}=\left((t, x)^{(\eta)}, p, w, q\right) \in \Omega_{H}^{(0)}$,

5) there is $M_{\phi} \in \mathbb{R}_{+}$such that

$$
\left|\phi_{h}^{(\eta)}-\bar{\phi}_{h}^{(\eta)}\right| \leq h_{0} M_{\phi} H_{h}^{(\eta)}, \quad(t, x)^{(\eta)} \in E_{0, h} .
$$

Then

$$
\left|v_{h}^{(\eta)}-u_{h}^{(\eta)}\right| \leq h_{0} \Psi\left(t^{(\eta)}\right) H_{h}^{(\eta)}, \quad(t, x)^{(\eta)} \in \widehat{E}_{h},
$$

where $\Psi:\left[-\tau_{0}, a\right] \rightarrow \mathbb{R}$ satisfies

$$
\begin{gathered}
\Psi(t) \geq M_{\phi}, \quad t \in\left[-\tau_{0}, 0\right], \\
\Psi^{\prime}(t)-L_{u}-B_{0}(h)-L_{1} \Psi(t) \\
-B_{1}(h, \psi(t)) \Gamma\left(\psi(t) \sqrt{1+\left(r_{1}(t)+\|\tau\|+\left\|h^{\prime}\right\|(\lambda+1)\right)^{2}}\right) \\
\times(\Gamma(\Psi(t) \widetilde{\theta}) \Gamma(\kappa(t) \widetilde{\theta}))^{-1} \geq 0
\end{gathered}
$$

for $t \in[0, a]$ and $h \in I_{d}$, where $\tilde{\theta}=\sqrt{1+\left(r_{0}(t, h)\right)^{2}}$, and $B_{0}$ and $B_{1}$ are defined by

$$
\begin{aligned}
B_{0}(h) & =\lambda^{2} L_{u}\left(h_{0}^{-1} \sum_{l=1}^{n} h_{l}\right)^{2}\left(\widehat{a}+\widehat{b} L_{2} h_{0}\left\|\left(h_{1}^{-1}, \ldots, h_{n}^{-1}\right)\right\|\right), \\
B_{1}(h, p) & =\bar{p} \widehat{p}\left(p+\lambda\left\|D_{t} u\right\|_{H}(a)+(\lambda+1) \sum_{l=1}^{n} h_{l} h_{0}^{-1}\left\|D_{x_{l}} u\right\|_{H}(a)\right)
\end{aligned}
$$

for $p \in \mathbb{R}_{+}$, and 


$$
\begin{aligned}
r_{0}(t, h) & =\frac{1+\sqrt{1+\left(\lambda\left\|h^{\prime}\right\| / h_{0}\right)^{2}}}{\psi^{\prime}(t) / \psi(t)} \lambda\left\|h^{\prime}\right\| / h_{0}, \\
r_{1}(t) & =\frac{\psi(t)\left(\|\tau\|+\left\|h^{\prime}\right\|(\lambda+1)\right)}{\kappa(t)-\psi(t)} .
\end{aligned}
$$

Moreover, we have

$$
\begin{aligned}
\Psi^{\prime}(t) & -L_{1}-L_{u}+\left(\Psi(t)-h_{0} L_{u}\right) \psi^{\prime}(t) \Gamma^{\prime}(\psi(t)) / \Gamma(\psi(t)) \\
& -B_{0}(h) \Gamma\left(\psi(t) \sqrt{1+\left(\left\|h^{\prime}\right\| \lambda / h_{0}+r_{0}(t, h)\right)^{2}}\right) / \Gamma(t) \\
& -B_{1}(h, \Psi(t)) \Gamma\left(\psi(t) \sqrt{1+\left(r_{0}(t, h)+\|\tau\|+\left\|\bar{h}^{\prime}\right\|(1+\lambda)\right)^{2}}\right) \\
& \times(\Gamma(\kappa(t)) \Gamma(\psi(t)))^{-1} \geq 0
\end{aligned}
$$

for $t \in[0, a]$ with $\Psi(t)$ so large that $\Psi(t)-\bar{h}_{0} L_{u} \geq 0$.

Proof. Let $w_{h}^{(\eta)}=u_{h}^{(\eta)}-v_{h}^{(\eta)}$ for $(t, x)^{(\eta)} \in \widehat{E}_{h}$. From (2.5) we obtain the recurrence equality

$$
\begin{aligned}
w_{h}^{\left(\eta_{0}+1, \eta^{\prime}\right)}= & A w_{h}^{(\eta)} \\
& +h_{0}\left(f\left((t, x)^{(\eta)}, u_{h}^{(\eta)},\left(\left[u_{h}\right]_{h}\right)_{(\eta)}, \Delta u_{h}^{(\eta)}\right)\right. \\
& \left.-f\left((t, x)^{(\eta)}, u_{h}^{(\eta)},\left(\left[u_{h}\right]_{h}\right)_{(\eta)}, \Delta v_{h}^{(\eta)}\right)\right) \\
& +h_{0}\left(f\left((t, x)^{(\eta)}, u_{h}^{(\eta)},\left(\left[u_{h}\right]_{h}\right)_{(\eta)}, \Delta v_{h}^{(\eta)}\right)\right. \\
& \left.-f\left((t, x)^{(\eta)}, v_{h}^{(\eta)},\left(\left[v_{h}\right]_{h}\right)_{(\eta)}, \Delta v_{h}^{(\eta)}\right)\right) \\
& +h_{0}\left(\Delta_{0} u_{h}^{(\eta)}-f\left((t, x)^{(\eta)}, u_{h}^{(\eta)},\left(\left[u_{h}\right]_{h}\right)_{(\eta)}, \Delta u_{h}^{(\eta)}\right)\right)
\end{aligned}
$$

for $(t, x)^{(\eta)} \in E_{h}$. Using the mean value theorem, the Lipschitz condition for $f$, and Lemma 2.1, we obtain the recurrence estimate

$$
\begin{aligned}
\left|w_{h}^{\left(\eta_{0}+1, \eta^{\prime}\right)}\right| \leq & \sum_{s \in S_{\lambda}}\left|w_{h}^{\left(\eta_{0}, \eta^{\prime}+s\right)}\right|\left|a_{s}^{(\eta)}+h_{0} \sum_{l=1}^{n} h_{l}^{-1} b_{s, l}^{(\eta)} D_{q_{l}} f\left(P^{(\eta)}\right)\right| \\
& +h_{0} L_{1}\left|w_{h}^{(\eta)}\right|+h_{0} \mathcal{L}\left((t, x)^{(\eta)}\right)\left\|\left(\left[w_{h}\right]_{h}\right)_{(\eta)}\right\|_{D}+h_{0} \mu_{h}^{(\eta)}
\end{aligned}
$$

for $(t, x)^{(\eta)} \in E_{h}$. Now, using (3.1) and Assumption $H_{1}$, we easily obtain from (3.11) the following inequality which is much easier to analyse:

$$
\begin{aligned}
\left|w_{h}^{\left(\eta_{0}+1, \eta^{\prime}\right)}\right| \leq & \max _{s \in S_{\lambda}}\left|w_{h}^{\left(\eta_{0}, \eta^{\prime}+s\right)}\right|+h_{0} L_{1}\left|w_{h}^{(\eta)}\right| \\
& +h_{0} \mathcal{L}\left((t, x)^{(\eta)}\right)\left\|\left(\left[w_{h}\right]_{h}\right)_{(\eta)}\right\|_{D}+h_{0} \mu_{h}^{(\eta)}
\end{aligned}
$$

for $(t, x)^{(\eta)} \in E_{h}$. If we prove that the function $\mathcal{W}_{h}^{(\eta)}=h_{0} \Psi\left(t^{(\eta)}\right) H_{h}^{(\eta)}$, $(t, x)^{(\eta)} \in \widehat{E}_{h}$, satisfies a comparison inequality with respect to (3.12), then 
(3.3) will be established. Thus, in order to finish the proof of our theorem it is enough to prove the following

Lemma 3.1. If the assumptions of Theorem 3.1 are satisfied, then

$$
\begin{aligned}
\mathcal{W}_{h}^{\left(\eta_{0}+1, \eta^{\prime}\right)} \geq & \mathcal{W}_{h}^{\left(\eta_{0},\left|\eta^{\prime}\right|+\lambda^{\prime}\right)}+h_{0} L_{1} \mathcal{W}_{h}^{(\eta)} \\
& +h_{0} \mathcal{L}\left((t, x)^{(\eta)}\right)\left\|\left(\left[\mathcal{W}_{h}\right]_{h}\right)_{(\eta)}\right\|_{D}+h_{0} \mu_{h}^{(\eta)}
\end{aligned}
$$

for $(t, x)^{(\eta)} \in E_{h}$, and

$$
\mathcal{W}_{h}^{(\eta)} \geq h_{0} M_{\phi} H_{h}^{(\eta)}, \quad(t, x)^{(\eta)} \in E_{0, h} .
$$

P r o o f. Condition (3.14) follows immediately from (3.4) and (3.2). Condition (3.13) is a consequence of

$$
\begin{aligned}
(\Psi(t+ & \left.\left.h_{0}\right)-h_{0} L_{u}\right) \Gamma\left(\psi\left(t+h_{0}\right) \sqrt{1+r^{2}}\right) \\
\geq & \left.\Psi(t)+h_{0} B_{0}(h)\right) \Gamma\left(\psi(t) \sqrt{1+\left(r+\left\|h^{\prime}\right\| \lambda\right)^{2}}\right) \\
& +h_{0} B_{1}(h, \Psi(t)) \Gamma\left(\psi(t) \sqrt{1+\left(r+\|\tau\|+\left\|h^{\prime}\right\|(\lambda+1)\right)^{2}}\right) \\
& \times\left(\Gamma\left(\psi(t) \sqrt{1+r^{2}}\right)\right)^{-1}
\end{aligned}
$$

for $t \in\left[0, a-h_{0}\right]$ and $r=\|x\| \in \mathbb{R}_{+}$, where $B_{0}(h)$ and $B_{1}(h, \Psi(t))$ are given by (3.6) and (3.7). This implication follows from (3.9).

If $r$ is greater than $r_{0}(t, h)$ given by (3.8), then

$$
\psi\left(t+h_{0}\right) \sqrt{1+r^{2}} \geq \psi(t) \sqrt{1+\left(r+\left\|h^{\prime}\right\| \lambda\right)^{2}},
$$

and (3.15) follows from

$$
\begin{aligned}
\Xi(\theta):= & \Psi(t+\theta)-\theta L_{u}-\Psi(t)-\theta B_{0}(h)-\theta L_{1} \Psi(t) \\
& -\theta B_{1}(h, \Psi(t)) \Gamma\left(\psi(t) \sqrt{1+\left(r_{1}(t)+\|\tau\|+\left\|h^{\prime}\right\|(\lambda+1)\right)^{2}}\right) \\
& \times(\Gamma(\psi(t) \widetilde{\theta}) \Gamma(\kappa(t) \widetilde{\theta}))^{-1} \geq 0,
\end{aligned}
$$

where $\theta \in\left[0, h_{0}\right], t \in\left[0, a-h_{0}\right]$ and $\widetilde{\theta}$ is the same as in (3.5). Now, (3.17) holds true because $\Xi(0)=0$ and $\Xi^{\prime}(\theta) \geq 0$ for $\theta \in\left[0, h_{0}\right]$ as we have (3.5).

For $r \leq r_{0}\left(x_{0}, h\right)$ and $t \in\left[0, a-h_{0}\right]$, formula (3.15) is a consequence of the inequality $\Xi_{1}(\theta ; t, r) \geq 0$ for $\theta \in\left[0, h_{0}\right]$, where

$$
\begin{aligned}
& \Xi_{1}(\theta ; t, r) \\
:= & \left(\Psi(t+\theta)-\theta L_{u}\right) \Gamma\left(\psi(t+\theta) \sqrt{1+r^{2}}\right)-\theta L_{1} \Gamma\left(\psi(t) \sqrt{1+r^{2}}\right) \\
& -\left(\Psi(t)+\theta B_{0}(h)\right) \Gamma\left(\psi(t) \sqrt{1+\left(r+\theta \lambda\left\|h^{\prime}\right\| / h_{0}\right)^{2}}\right) \\
- & \theta B_{1}(h, \Psi(t)) \Gamma\left(\psi(t) \sqrt{1+\left(r_{0}(t, h)+\|\tau\|+\left\|\overline{h^{\prime}}\right\|(\lambda+1)\right)^{2}}\right) / \Gamma(\kappa(t))
\end{aligned}
$$


for $\theta \in\left[0, h_{0}\right]$ and $t \in\left[0, a-h_{0}\right]$. From (3.18) we find a lower estimate of $\Xi_{1}^{\prime}(\theta ; t, h)$ :

$$
\begin{aligned}
& \Xi_{1}^{\prime}(\theta ; t, h) \\
\geq & \left(\Psi^{\prime}(t+\theta)-L_{u}\right) \Gamma\left(\psi(t+\theta) \sqrt{1+r^{2}}\right) \\
+ & \left(\Psi(t+\theta)-\theta L_{u}\right) \Gamma^{\prime}\left(\psi(t+\theta) \sqrt{1+r^{2}}\right) \psi^{\prime}(t+\theta) \sqrt{1+r^{2}} \\
- & L_{1} \Gamma\left(\psi(t) \sqrt{1+r^{2}}\right)-B_{0}(h) \Gamma\left(\psi(t) \sqrt{1+\left(r+\left\|h^{\prime}\right\| \lambda\right)^{2}}\right) \\
- & \left(\Psi(t)+\theta B_{0}(h)\right) \Gamma^{\prime}\left(\psi(t) \sqrt{1+\left(r+\lambda\left\|H^{\prime}\right\|\right)^{2}}\right) \psi^{\prime}(t) \\
& -B_{1}(h, \Psi(t)) \Gamma\left(\psi(t) \sqrt{1+\left(r_{0}(t, h)+\|\tau\|+\bar{h}^{\prime} \|(\lambda+1)\right)^{2}}\right) / \Gamma(\kappa(t)) .
\end{aligned}
$$

From (3.19) and (3.9) we obtain $\Xi^{\prime}(\theta ; t, h) \geq 0$, and (3.18) implies $\Xi(0 ; t, h)=0$. Therefore, $\Xi\left(h_{0} ; t, h\right) \geq 0$ for $t \in\left[0, a-h_{0}\right]$. This completes the proof.

R e mark. Condition 1) of Theorem 3.1 can be much weaker, namely $\mathcal{L}$ might be defined by

$$
\mathcal{L}(t, x)=\Gamma\left(\psi(t) \sqrt{1+\|x\|^{2}}\right) / \Gamma\left(\kappa(t) \sqrt{1+\|x\|^{2}}\right), \quad(t, x) \in E .
$$

In this case the function $\Psi$ satisfies stronger conditions than (3.4), (3.5) and (3.9).

If $\Gamma(t) \geq$ const $e_{2}(t)$, then $\mathcal{L}$ might be defined by

$$
\mathcal{L}(t, x)=\left(\Gamma\left(\psi(t) \sqrt{1+\|x\|^{2}}\right)\right)^{\nu} / \Gamma\left(\kappa(t) \sqrt{1+\|x\|^{2}}\right), \quad(t, x) \in E,
$$

where $\nu \geq 0$, and the proof works for a sufficiently large function $\Psi$.

4. Convergence result for another functional dependence. If $L, L_{1}, L_{2} \in \mathbb{R}_{+}$, then $f$ is of class $\operatorname{Lip}\left(\Omega_{H}^{(1)} ; L, L_{1}, L_{2}\right)$ iff $f \in C\left(\Omega_{H}^{(1)}, \mathbb{R}\right)$ and

$$
\begin{aligned}
\mid f(t, x, p, w, q)- & f(t, x, \bar{p}, \bar{w}, \bar{q}) \mid \\
\leq & L_{1}|p-\bar{p}|+L H(t, x)\|w-\bar{w}\|_{H}(t)+L_{2}\|q-\bar{q}\|
\end{aligned}
$$

for all $(t, x, p, w, q),(t, x, \bar{p}, \bar{w}, \bar{q}) \in \Omega_{H}^{(1)}$. For $f \in C\left(\Omega_{H}^{(1)}, \mathbb{R}\right)$ we consider the Cauchy problem for the equation

$$
D_{t} z(t, x)=f\left(t, x, z(t, x), z, D_{x} z(t, x)\right) .
$$

We assume that the Cauchy problem (4.2), (1.4) has a unique solution of class $\mathcal{C}_{H}$ defined on $E_{0} \cup E$.

Let $f \in \mathcal{C}_{H}\left(\Omega_{H}^{(1)}\right)$. Then the difference analogue of (4.2) reads

$$
\Delta_{0} z_{h}^{(\eta)}=f\left((t, x)^{(\eta)}, z_{h}^{(\eta)},\left[z_{h}\right]_{h}, \Delta z_{h}^{(\eta)}\right), \quad(t, x)^{(\eta)} \in E_{h} .
$$

Problem (4.3) is also considered with initial condition (2.6). 
Lemma 4.1. Suppose that Assumptions $H_{1}$ and $H_{2}$ are satisfied and $H \in \mathcal{H}, \varepsilon \in \mathcal{E}_{H}$. Let $f \in \mathcal{C}_{H}\left(\Omega_{H}^{(1)}\right)$ with constants $L, L_{0}, L_{1}, L_{2} \in \mathbb{R}_{+}$. Let $u \in C\left(E_{0} \cup E, \mathbb{R}\right)$ be a solution of $(4.2)$, (1.4) such that $u, D_{t} u, D_{x_{l}} u \in \mathcal{C}_{H, \varepsilon}$ for $l=1, \ldots, n$, and there is $L_{u} \in \mathbb{R}_{+}$such that

$$
\begin{aligned}
& \left|D_{x_{l}}(t, x)-D_{x_{l}} u(\bar{t}, \bar{x})\right|+\left|D_{t} u(t, x)-D_{t} u(\bar{t}, \bar{x})\right| \\
& \quad \leq L_{u}\left(|t-\bar{t}|+\sum_{j=1}^{n}\left|x_{j}-\bar{x}_{j}\right|\right) \max \{H(t, x) \varepsilon(t, x), H(\bar{t}, \bar{x}) \varepsilon(\bar{t}, \bar{x})\}
\end{aligned}
$$

for $(t, x),(\bar{t}, \bar{x}) \in E$ and $l=1, \ldots, n$. Assume also that for $h \in I_{d}$ and $s \in S_{\lambda+1}$ there is $R(h) \in \mathbb{R}_{+}$such that $\lim \sup \left\{R(h) \mid h \in I_{d}\right\}<\infty$, and

$$
\sum_{l=1}^{n} x_{l} D_{x_{l}}\left(\widetilde{H}\left(t+h_{0}, x+\left(x^{(s)}\right)\right) / H(t, x)\right) \leq 0
$$

for $\|x\| \geq R(h)$ and $\left(t+h_{0}, x\right) \in E$, where

$$
\widetilde{H}(t, x)=H(t, x) \varepsilon(t, x), \quad(t, x) \in E .
$$

Then

$$
\left|\Delta_{0} u_{h}^{(\eta)}-f\left((t, x)^{(\eta)}, u_{h}^{(\eta)},\left[u_{h}\right]_{h}, \Delta u_{h}^{(\eta)}\right)\right| \leq \mu_{h}^{(\eta)},
$$

where

$$
\begin{aligned}
\mu_{h}^{(\eta)}= & h_{0} L_{u} \widetilde{H}_{h}^{\left(\eta_{0}+1, \eta^{\prime}\right)}+h_{0}^{-1} \widehat{a} \lambda^{2}\left(\sum_{l=1}^{n} h_{l}\right)^{2} L_{u} \widetilde{H}_{h}^{\left(\eta_{0},\left|\eta^{\prime}\right|+\lambda^{\prime}\right)} \\
& +L \widetilde{H}_{h}^{(\eta)} \widehat{p}\left(h_{0} \lambda\left\|D_{t} u\right\|_{H}(a)+(\lambda+1) \sum_{l=1}^{n} h_{l}\left\|D_{x_{l}} u\right\|_{H}(a)\right) \\
& \times \sup _{\bar{\eta}} \sup _{x^{(\bar{\eta}-1)}<\bar{x} \leq x} \frac{\left.\widetilde{H}_{h}^{(\bar{\eta})},\left|\bar{\eta}_{0},\right| \bar{\eta}^{\prime} \mid+\lambda^{\prime}\right)}{H(\bar{t}, \bar{x})} \\
& +L_{2}\left\|\left(h_{1}^{-1}, \ldots, h_{n}^{-1}\right)\right\| \widehat{b}\left(\sum_{l=1}^{n} h_{l}\right)^{2} \lambda^{2} L_{u} \widetilde{H}_{h}^{\left(\eta_{0},\left|\eta^{\prime}\right|+\lambda^{\prime}\right)},
\end{aligned}
$$

where $(t, x)^{(\eta)} \in E_{h}$, and $\widetilde{H}$ is defined by (4.6).

Remark. Condition (4.5) is satisfied if, for example, $H$ is defined by (1.5), and

$$
\varepsilon(t, x)=\Gamma\left(\xi(t) \sqrt{1+\|x\|^{2}}\right) / \Gamma\left(\psi(t) \sqrt{1+\|x\|^{2}}\right),
$$

where $\xi \in C^{1}\left(\left[-\tau_{0}, a\right], \mathbb{R}_{+}\right)$is increasing, and $0<\xi(t)<\psi(t)$ for $t \in\left[-\tau_{0}, a\right]$. We should only assume that $\bar{h}_{0}<(\psi(t)-\xi(t)) / \xi^{\prime}(a)$ for $t \in\left[-\tau_{0}, a\right]$. 
Proof of Lemma 4.1. Estimates (2.13)-(2.16) remain true if we replace $H$ by $\widetilde{H}$ defined by (4.6). Thus, in order to obtain (4.7) with $\mu_{h}$ defined by (4.8), it is sufficient to estimate $L H_{h}^{(\eta)}\left\|\left[u_{h}\right]_{h}-u\right\|_{H}(a)$ by a suitably chosen expression basing on formula (2.16) with $H$ replaced by $\widetilde{H}$. The double supremum appearing in (4.8) is finite because (4.5) guarantees that $\widetilde{H}_{h}^{\left(\bar{\eta}_{0},\left|\bar{\eta}^{\prime}\right|+\lambda^{\prime}\right)} / H(\bar{t}, \bar{x})$ is bounded by

$$
M_{h}=\sup \left\{\begin{array}{l|l}
\widetilde{H}\left(t+h_{0}, x+\left(x^{(s)}\right)\right) / H(t, x) & \begin{array}{l}
\|x\| \leq R(h), \\
\left(t+h_{0}, x\right) \in E, \\
s \in S_{\lambda+1}
\end{array}
\end{array}\right\},
$$

where $h \in I_{d}$. The rest works as in the proof of Lemma 2.1.

Theorem 4.1. Suppose that

1) Assumptions $H_{1}$ and $H_{2}$ are satisfied,

2) $H \in \mathcal{H}$ and $\varepsilon \in \mathcal{E}_{H}$ are given by (1.5) and (4.9), respectively, where $\xi \in C\left(\left[-\tau_{0}, a\right], \mathbb{R}_{+}\right)$has positive derivative on $[0, a] ; 0<\xi(t)<\psi(t)$ for $t \in\left[-\tau_{0}, a\right] ; \widetilde{H} \in \mathcal{H}$ is defined by (4.6), and $f \in \mathcal{C}_{H}\left(\Omega_{H}^{(1)}\right)$ with constants $L$, $L_{1}, L_{2}$,

3) $u \in C\left(E_{0} \cup E, \mathbb{R}\right)$ is a solution of (4.2), (1.4) such that $u, D_{t} u, D_{x_{l}} u \in$ $\mathcal{C}_{H, \varepsilon}$ for $l=1, \ldots, n$, and there exists $L_{u} \in \mathbb{R}_{+}$such that condition (4.4) is satisfied, and the constants $M_{h}$ defined by (4.10) for $h \in I_{d}$ are such that $\lim \sup \left\{M_{h} \mid h \in I_{d}\right\} \leq M$,

4) $v_{h} \in \mathcal{F}_{H, \varepsilon}^{(h)}$ is a solution of (4.3), (2.6),

5) the monotonicity condition (3.1) is satisfied for $s \in S_{\lambda},(t, x)^{(\eta)} \in E_{h}$ and $P^{(\eta)}=\left((t, x)^{(\eta)}, p, z, q\right) \in \Omega_{H}^{(1)}$,

6) there is $M_{\phi} \in \mathbb{R}$ such that

$$
\left|\phi_{h}^{(\eta)}-\bar{\phi}_{h}^{(\eta)}\right| \leq h_{0} M_{\phi} \widetilde{H}_{h}^{(\eta)}, \quad(t, x)^{(\eta)} \in E_{h},
$$

7) $\bar{h}_{0}$ is so small that $\psi(t)-\xi(t)-\bar{h}_{0} \xi^{\prime}(t)>0$ for $t \in[0, a]$.

Then

$$
\left|v_{h}^{(\eta)}-u_{h}^{(\eta)}\right| \leq h_{0} \Psi\left(t^{(\eta)}\right) \widetilde{H}_{h}^{(\eta)}, \quad(t, x)^{(\eta)} \in \widehat{E}_{h},
$$

where $\Psi:\left[-\tau_{0}, a\right] \rightarrow \mathbb{R}_{+}$satisfies inequality (3.4) and

$$
\begin{aligned}
& \Psi^{\prime}(\theta)-L_{u}-L_{1}-\widetilde{B}_{0}(h) \\
& \quad-\widetilde{B}(h, \Psi(\theta)) \Gamma\left(\xi(\theta) \sqrt{1+\left(r_{1}(t)\right)^{2}}\right) / \Gamma(\psi(0)) \geq 0
\end{aligned}
$$

for $\theta \in[0, a]$, where $\widetilde{B}_{0}(h), r_{1}(t)$ and $\widetilde{B}(h, p)$ for $p \in \mathbb{R}_{+}$are defined by 


$$
\begin{aligned}
\widetilde{B}_{0}(h) & =\lambda^{2} L_{u}\left(\sum_{l=1}^{n} h_{l} / h_{0}\right)^{2}\left(\widehat{a}+L_{2} \widehat{b}\left\|\left(h_{0} h_{1}^{-1}, \ldots, h_{0} h_{n}^{-1}\right)\right\|\right), \\
\widetilde{B}(h, p) & =L \widehat{p}\left(\lambda\left\|D_{t} u\right\|_{H}(a)+(\lambda+1) \sum_{l=1}^{n} h_{l} h_{0}^{-1}\left\|D_{x_{l}} u\right\|_{H}(a)+p\right), \\
r_{1}(t) & =(\lambda+1)\left\|\bar{h}^{\prime}\right\|\left(1+\psi(t)\left(\psi(t)-\xi(t)-\bar{h}_{0} \xi^{\prime}(t)\right)^{-1}\right),
\end{aligned}
$$

where $\theta, t \in[0, a], h \in I_{d}$, and

$$
\begin{aligned}
\left(\Psi^{\prime}(\theta)\right. & \left.-L_{u}\right) \Gamma(\xi(\theta))+\Gamma^{\prime}(\xi(\theta)) \xi^{\prime}(\theta)\left(\Psi(\theta)-h_{0} L_{u}\right) \\
& -\Gamma\left(\xi(\theta) \sqrt{1+\left(r_{0}(t)\right)^{2}}\right) \\
& \times\left(L_{1} \Psi(\theta)+\widetilde{B}(h, \Psi(t)) \Gamma\left(\xi(\theta) \sqrt{1+\left(r_{1}(\theta)\right)^{2}}\right) / \Gamma(\psi(0))\right) \\
& -\left(\Psi(\theta)+h_{0} \widetilde{B}_{0}(h)\right) \\
& \times \Gamma^{\prime}\left(\xi(\theta) \sqrt{1+\left(r_{0}(\theta)+\lambda\left\|h^{\prime}\right\|\right)^{2}}\right) \xi(\theta) \lambda\left\|h^{\prime}\right\| / h_{0} \\
& -\widetilde{B}_{0}(h) \Gamma\left(\xi(\theta) \sqrt{1+\left(r_{0}(\theta)+\lambda\left\|h^{\prime}\right\|\right)^{2}}\right) \geq 0
\end{aligned}
$$

for $\theta \in[0, a], h \in I_{d}$ with $\bar{h}_{0}$ so small that $\Psi(\theta)-\bar{h}_{0} L_{u} \geq 0$ on $\left[-\tau_{0}, a\right]$.

Proof. Let $w_{h}^{(\eta)}=u_{h}^{(\eta)}-v_{h}^{(\eta)}$ for $(t, x)^{(\eta)} \in \widehat{E}_{h}$. From (4.11) it follows that (4.12) is satisfied for $(t, x)^{(\eta)} \in E_{0, h}$. Subtracting the recurrence expressions of $v_{h}^{\left(\eta_{0}+1, \eta^{\prime}\right)}$ from $u_{h}^{\left(\eta_{0}+1, \eta^{\prime}\right)}$ leads to the recurrence error estimates similar to (3.12), as in the proof of Theorem 3.1 (compare (3.10) and (3.11)):

$$
\begin{aligned}
\left|w_{h}^{\left(\eta+1, \eta^{\prime}\right)}\right| \leq & \max _{s \in S_{\lambda}}\left|w_{h}^{\left(\eta_{0}, \eta^{\prime}+s\right)}\right|+h_{0} L_{1}\left|w_{h}^{(\eta)}\right| \\
& +h_{0} L \widetilde{H}_{h}^{(\eta)}\left\|\left[w_{h}\right]_{h}\right\|_{H}(a)+h_{0} \mu_{h}^{(\eta)}
\end{aligned}
$$

for $(t, x)^{(\eta)} \in E_{h}$, where $\mu_{h}$ is defined by (4.8). In order to establish (4.12) on $\widehat{E}_{h}$ it is sufficient to prove the following

LEMMA 4.2. If the assumptions of Theorem 4.1 are satisfied, then the function $\widetilde{\mathcal{W}}_{h}: \widehat{E}_{h} \rightarrow \mathbb{R}_{+}$given by $\widetilde{\mathcal{W}}_{h}^{(\eta)}=h_{0} \Psi\left(t^{(\eta)}\right) \widetilde{H}_{h}^{(\eta)}$ for $(t, x)^{(\eta)} \in \widehat{E}_{h}$ satisfies the inequality

$$
\begin{aligned}
\left|\widetilde{\mathcal{W}}_{h}^{\left(\eta_{0}+1, \eta^{\prime}\right)}\right| \geq \widetilde{\mathcal{W}}_{h}^{\left(\eta_{0},\left|\eta^{\prime}\right|+\lambda^{\prime}\right)}+h_{0} L_{1}\left|\widetilde{\mathcal{W}}_{h}^{(\eta)}\right| \\
+h_{0} L \widetilde{H}_{h}^{(\eta)}\left\|\left[\widetilde{\mathcal{W}}_{h}\right]_{h}\right\|_{H}(a)+h_{0} \mu_{h}^{(\eta)}
\end{aligned}
$$

for $(t, x)^{(\eta)} \in E_{h}$, and

$$
\widetilde{\mathcal{W}}_{h}^{(\eta)} \geq h_{0} M_{\phi} \widetilde{H}_{h}^{(\eta)}, \quad(t, x)^{(\eta)} \in E_{0, h} .
$$


Proof. Inequality (4.18) is obvious. Formula (4.17) follows from

$$
\begin{aligned}
& \left(\Psi\left(t+h_{0}\right)-h_{0} L_{u}\right) \Gamma\left(\xi\left(t+h_{0}\right) \sqrt{1+r^{2}}\right) \\
& \geq h_{0} L_{1} \Psi(t) \Gamma\left(\xi(t) \sqrt{1+r^{2}}\right)+h_{0} \widetilde{B}(h, \Psi(t))
\end{aligned}
$$

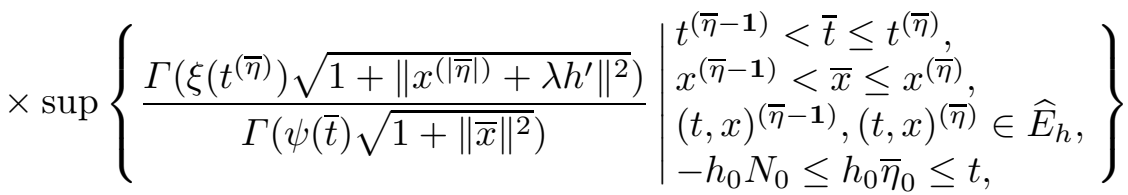

$$
\begin{aligned}
& +\left(\Psi(t)+h_{0} \widetilde{B}_{0}(h)\right) \Gamma\left(\xi(t) \sqrt{1+\left(r+\lambda\left\|h^{\prime}\right\|\right)^{2}}\right)
\end{aligned}
$$

for $t \in\left[0, a-h_{0}\right], r \in \mathbb{R}_{+}$and $h=\left(h_{0}, h^{\prime}\right) \in I_{d}$.

First, observe that

$$
\begin{aligned}
& \Gamma\left(\xi\left(t^{(\bar{\eta})}\right) \sqrt{1+\left\|x^{(|\bar{\eta}|)}+\lambda h^{\prime}\right\|^{2}}\right) / \Gamma\left(\psi(\bar{t}) \sqrt{1+\|\bar{x}\|^{2}}\right) \\
& \leq \Gamma\left(\xi\left(t^{(\eta)}\right) \sqrt{1+\left(r_{1}(t)\right)^{2}}\right) / \Gamma\left(\psi(\bar{t}) \sqrt{1+\|\bar{x}\|^{2}}\right)
\end{aligned}
$$

for $t^{(\bar{\eta}-\mathbf{1})}<\bar{t} \leq t^{(\bar{\eta})}, x^{(\bar{\eta}-\mathbf{1})}<\bar{x} \leq x^{(\bar{\eta})},(t, x)^{(\bar{\eta}-\mathbf{1})},(t, x)^{(\bar{\eta})} \in \widehat{E}_{h},-N_{0} \leq$ $\bar{\eta}_{0} \leq \eta_{0}$. We claim that

$$
\begin{aligned}
\widetilde{\Xi}(\theta ; t):= & \Psi(t+\theta)-\Psi(t)-\theta\left(L_{u}+L_{1}+\widetilde{B}_{0}(h)\right. \\
& \left.+\widetilde{B}(h, \Psi(t)) \Gamma\left(\xi(t) \sqrt{1+\left(r_{1}(t)\right)^{2}}\right) / \Gamma(\psi(0))\right) \geq 0
\end{aligned}
$$

for $t \in\left[0, a-h_{0}\right]$ and $\theta \in\left[0, h_{0}\right]$, because $\widetilde{\Xi}(0 ; t)=0$ and $\widetilde{\Xi}^{\prime}(\theta ; t) \geq 0$ on the considered interval as we have (4.13). Thus, if $r \geq r_{0}(t)$, then (4.19) results from (4.20) and (4.21).

If $r \leq r_{0}(t)$, then we define

$$
\begin{aligned}
\widetilde{\Xi}_{1}(\theta ; t, r)= & \left(\Psi(t+\theta)-\theta L_{u}\right) \Gamma\left(\xi(t+\theta) \sqrt{1+r^{2}}\right) \\
& -\theta \Gamma\left(\xi(t) \sqrt{1+r^{2}}\right)\left(L_{1} \Psi(t)\right. \\
& \left.+\widetilde{B}(h, \Psi(T)) \Gamma\left(\xi(t) \sqrt{1+\left(r_{1}(t)\right)^{2}}\right) / \Gamma(\psi(0))\right) \\
& -\left(\Psi(t)+\theta \widetilde{B}_{0}(h)\right) \Gamma\left(\xi(t) \sqrt{1+\left(r+\theta \lambda\left\|h^{\prime}\right\| / h_{0}\right)^{2}}\right)
\end{aligned}
$$

for $t \in\left[0, a-h_{0}\right]$ and $\theta \in\left[0, h_{0}\right]$. Next, from (4.15) we have

$$
\begin{aligned}
(4.23) & \widetilde{\Xi}_{1}^{\prime}(\theta ; t, r) \\
= & \left(\Psi^{\prime}(t)-L_{u}\right) \Gamma\left(\xi(t+\theta) \sqrt{1+r^{2}}\right) \\
& +\Gamma^{\prime}\left(\xi(t+\theta) \sqrt{1+r^{2}}\right) \xi^{\prime}(t+\theta) \sqrt{1+r^{2}}\left(\Psi(t+\theta)-\theta L_{u}\right) \\
& -\Gamma\left(\xi(t) \sqrt{1+r^{2}}\right)\left(L_{1} \Psi(t)+\widetilde{B}(h, \Psi(t)) \Gamma\left(\xi(t) \sqrt{1+\left(r_{1}(t)\right)^{2}}\right) / \Gamma(\psi(0))\right) \\
& -\left(\Psi(t)+\theta \widetilde{B}_{0}(h)\right) \Gamma^{\prime}\left(\xi(t) \sqrt{1+\left(r+\theta \lambda\left\|h^{\prime}\right\| / h_{0}\right)^{2}}\right) \psi(t) \lambda\left\|h^{\prime}\right\| / h_{0} \\
& -\widetilde{B}_{0}(h) \Gamma\left(\xi(t) \sqrt{1+\left(r+\theta \lambda\left\|h^{\prime}\right\| / h_{0}\right)^{2}}\right) \geq 0
\end{aligned}
$$


for $t \in\left[0, a-h_{0}\right]$ and $\theta \in\left[0, h_{0}\right]$. From $(4.21)$ it is easy to obtain $\widetilde{\Xi}_{1}(0 ; t, r)=$ 0 . From this and (4.23) we have $\widetilde{\Xi}_{1}\left(h_{0} ; t, r\right) \geq 0$. This finishes the proof of our lemma.

\section{References}

[1] P. Besala, On solutions of first order partial differential equations defined in an unbounded zone, Bull. Acad. Polon. Sci. 12 (1964), 95-99.

[2] - Finite difference approximation to the Cauchy problem for non-linear parabolic differential equations, Ann. Polon. Math. 46 (1985), 19-26.

[3] Z. Kamont, On the Cauchy problem for system of first order partial differential equations, Serdica 5 (1979), 327-339.

[4] M. Krzyżański, Partial Differential Equations of Second Order, PWN, Warszawa, 1971.

[5] H. Leszczyński, General finite difference approximation to the Cauchy problem for non-linear parabolic differential-functional equations, Ann. Polon. Math. 53 (1991), $15-28$.

[6] - Uniqueness results for unbounded solutions of first order non-linear differentialfunctional equations, Acta Math. Hungar. 64 (1994), 75-92.

[7] M. Malec et A. Schiaffino, Méthode aux différences finies pour une équation nonlinéaire différentielle fonctionnelle du type parabolique avec une condition initiale de Cauchy, Boll. Un. Mat. Ital. (7) 1-B (1987), 99-109.

[8] K. Prządka, Difference methods for non-linear partial differential functional equations of the first order, Math. Nachr. 138 (1988), 105-123.

[9] J. Szarski, Differential Inequalities, PWN, Warszawa, 1967.

Institute of Mathematics

University of Gdańsk

57 Wita Stwosza St.

80-952 Gdańsk, Poland

E-mail: hleszcz@ksinet.univ.gda.pl 